\title{
SEMIGROUPS WITH FEW ENDOMORPHISMS
}

\author{
IN MEMORIAM ANATOLIĬ IVANOVIC MAL'GEV
}

VLASTIMIL DLAB and B. H. NEUMANN

(Received 2 February 1968)

\section{Introduction}

Large finite groups have large automorphism groups [4]; infinite groups may, like the infinite cyclic group, have finite automorphism groups, but their endomorphism semigroups are infinite (see Baer [1, p. 530] or $[2, \mathrm{p}$. $68]$ ). We show in this paper that the corresponding propositions for semigroups are false.

\section{Finite semigroups ${ }^{1}$}

Consider the semigroup given by the presentation (see [5] for the notation)

$$
E_{n}=\operatorname{sg} p\left(e, f ; e^{2}=e, f^{2}=f, e(f e)^{n}=(f e)^{n}=(f e)^{n} f\right),
$$

where $n$ is a positive integer at our disposal. We write 0 for $(j e)^{n}$, because it clearly is a zero. The elements of $E_{n}$ can be written in one of the forms

$$
e, f, \text { ef, fe, efe, } f e f, \cdots,(f e)^{n-1} f,(e f)^{n}, 0 \text {; }
$$

hence the order of the semigroup is

$$
\left|E_{n}\right| \leqq 4 n \text {. }
$$

To show that the order of $E_{n}$ in fact equals $4 n$, we represent the semigroup by mappings of the set

$$
X=\left\{1, e, f, \text { ef }, f e, \text { efe }, \cdots,(f e)^{n-1} f,(e f)^{n}, 0\right\}
$$

into itself. Define $\varepsilon: X \rightarrow X$ by

$$
\begin{aligned}
1 \varepsilon & =e \varepsilon=e, & & \\
(f e)^{i-1} f \varepsilon & =(f e)^{i} \varepsilon=(f e)^{i}, & & 1 \leqq i \leqq n-1 \\
(e f)^{i} \varepsilon & =(e f)^{i} e \varepsilon=(e f)^{i} e, & & 1 \leqq i \leqq n-1 \\
(f e)^{n-1} f \varepsilon & =(e f)^{n} \varepsilon=0 \varepsilon=0 ; & &
\end{aligned}
$$

1 We are indebted to Mr Bruce G. Neill, an undergraduate student at the University of Queensland, for several important corrections to an earlier draft of this section. 
and similarly $\zeta: X \rightarrow X$ by

$$
\begin{aligned}
1 \zeta & =f \zeta=f, & & \\
(e f)^{i-1} e \zeta & =(e f)^{i} \zeta=(e f)^{i}, & & 1 \leqq i \leqq n, \\
(f e)^{i} \zeta & =(f e)^{i} f \zeta=(f e)^{i} f, & & 1 \leqq i \leqq n-1, \\
0 \zeta & =0 . & &
\end{aligned}
$$

Then one can verify, firstly, that $\varepsilon$ and $\zeta$ satisfy the defining relations

$$
\varepsilon^{2}=\varepsilon, \zeta^{2}=\zeta, \varepsilon(\zeta \varepsilon)^{n}=(\zeta \varepsilon)^{n}=(\zeta \varepsilon)^{n} \zeta
$$

of $E_{n}$, and secondly that every element of $X$ other than 1 is the image of 1 under a sequence of mappings $\varepsilon$ and $\zeta$. It follows that the semigroup of mappings $X \rightarrow X$ generated by $\varepsilon$ and $\zeta$ is an epimorphic image of $E_{n}$ of order not less than $|X|-1$, that is $4 n$; thus it is isomorphic to $E_{n}$, and

$$
\left|E_{n}\right|=4 n \text {, }
$$

as claimed. This representation of $E_{n}$ is, of course, simply that by right multiplications on $E_{n}^{1}$, the semigroup obtained from $E_{n}$ by adjoining a unit element. Incidentally one sees that $E_{n}$ has only 3 idempotents, namely $e, f, 0$. As every endomorphism of $E_{n}$ must map $e$ and $f$ on idempotents, and as the images of $e$ and $f$ determine the endomorphism uniquely, there can be at most 9 endomorphisms of $E_{n}$. In fact there are only 8 , as the defining relations of $E_{n}$ are not symmetric in $e$ and $f$, and thus $e$ and $f$ cannot be interchanged by an endomorphism; the other 8 mappings of $e$ and $f$ independently on $e, f, 0$ all define endomorphisms. Thus we see that there are finite semigroups of arbitrarily large order with a bounded number of endomorphisms, namely 8.

It may be remarked that a finite semigroup has at least one idempotent, and thus must, if its order exceeds 1 , have at least 2 endomorphisms, namely the identity and one that maps all elements on one and the same idempotent. We do not know whether there are finite semigroups of arbitrarily large order with fewer than 8 endomorphisms.

\section{A rigid semigroup}

An infinite semigroup need not contain any idempotent, and it is thus conceivable that such a semigroup could be 'rigid', that is to say, have no endomorphisms other than the identity. We shall now exhibit a rigid semigroup.

THEOREM 3.1. The semigroup presented by

$$
A=\operatorname{sg} p\left(a, b ; a b^{2}=b a b a\right)
$$

has no endomorphism other than the identity automorphism. 
We defer the proof, which requires setting up a normal form for the elements of $A$ and then considering a number of cases. For the present we just remark that the theorem is best possible in more than one sense:

No monogenic semigroup, other than the trivial semigroup (of order 1), can be rigid; for every mapping of the generator of a monogenic semigroup into the semigroup extends to an endomorphism (or, equivalently, every monogenic semigroup, like every cyclic group, is relatively free). Thus a rigid semigroup needs, like ours, at least 2 elements to generate it.

A free semigroup has infinitely many endomorphisms, as every mapping of the generators into the semigroup extends to an endomorphism. Thus a rigid semigroup needs, like ours, at least one non-tautological relation.

The length of the defining relation of our semigroup $A$, that is to say the sum of the lengths of its two sides, is 7 . One can show that no semigroup with a defining relation of length 6 or less is rigid: the verification is tedious, and we omit it.

We have found other rigid semigroups with two generators and a single defining relation, of length $\mathbf{7}$ and also of greater lengths.

\section{A representation of the rigid semigroup}

Let $W$ denote the set of words, which we shall call normal words, in $a$ and $b$ of the form

$$
w==b^{t} a^{s_{1}} b a^{s_{2}} \cdots b a^{s_{n}}
$$

where

$$
\begin{aligned}
t & \geqq 0, \\
n & \geqq 1, \\
s_{n} & \geqq 0 \text { and } s_{i} \geqq 1 \text { for } 1 \leqq i<n .
\end{aligned}
$$

We shall sometimes omit zeroth powers of $a$ or $b$; thus if $t=0$ and $n=1$, we have a power $a^{s_{1}}$ of $a$; if $n=1$ and $s_{1}=0$ we have a power $b^{t}$ of $b$. The empty word is the normal word with $t=0, n=1$, and $s_{1}=0$; we denote it by 1 . Every normal word other than 1 represents ${ }^{2}$ an element of our semigroup

$$
A=\operatorname{sg} p\left(a, b ; a b^{2}=b a b a\right),
$$

or indeed of any other semigroup generated by $a$ and $b$.

Lemma 4.2. Every element of $A$ is represented by one and only one normal word.

2 A word is a string of symbols; if we interpret it as a product (which is written in the same way) we obtain an element of the semigroup, and we say the word represents the element. The same element of the semigroup can be represented by different words. 
Proof ${ }^{3}$. We use the method of Croisot as amplified by Clifford and Preston [3, pp. 169-171]. Our 'normal words' are their 'canonical forms' for $\rho^{*}$, the congruence on $F^{1}$ generated by the defining relation $\rho=\left\{\left(a b^{2}, b a b a\right)\right\}$; here $F^{1}$ is the free semigroup generated by $a, b$ with a unit element 1 adjoined. To apply the method of Clifford and Preston, we define a mapping

as follows.

$$
\psi: F^{1} \rightarrow F^{1}
$$

If $w \in F^{1}$ is of the form

$$
w=w^{\prime} a b^{2} w^{\prime \prime},
$$

we may assume this representation so chosen that $w^{\prime \prime}$ is of minimal length: thus $w^{\prime \prime}$ is not itself of the form (4.3). We then put

$$
w \psi=w^{\prime} b a b a w^{\prime \prime} .
$$

On the other hand, if $w$ is not of the form (4.3), we put

$$
w \psi=w .
$$

It is then clear that these latter words are just our normal words; and that to every $w \in F^{1}$ there is a non-negative integer $n$ such that

$$
w \psi^{n+1}=w \psi^{n} \in W .
$$

We define $\phi: F^{1} \rightarrow W$ by $w \phi=w \psi^{n}$ with $n$ chosen as in (4.4).

If we are given an arbitrary element of $A$, there is at least one word in $a$ and $b$, say $u$, that represents it. Then also $u \psi$ represents this element, and also $u \psi^{2}$, and ultimately also $u \phi$ : thus every element of $A$ is represented by at least one normal word.

To show the unicity we have to verify that $\psi$ has the properties (1), (2), and (3) of [3, p. 170], and that it implies (ii) of [3, p. 169]. Of these, (1), (2), and (ii) are obvious, and it only remains to verify that to each pair of elements $u, v$ in $F^{1}$ there are non-negative integers $k$ and $l$ such that

$$
\begin{aligned}
& (u v) \psi^{k+1}=((u \psi) v) \psi^{k}, \\
& (u v) \psi^{l+1}=(u(v \psi)) \psi^{l} .
\end{aligned}
$$

Now (4.52) is certainly satisfied if $l$ is chosen large enough, like $n$ in (4.4), to ensure that

$$
(u v) \psi^{l+1}=(u v) \psi^{l} .
$$

We turn to the existence of $k$ to satisfy (4.51). If $u \in W$, we choose $k$ so that

${ }^{3}$ [Added 1 May 1968.] We are indebted to the referee for suggesting a proof along these lines, shorter than our original proof. 


$$
(u v) \psi^{k+1}=(u v) \psi^{k},
$$

that is as $n$ in $(4.4)$; then $(4.51)$ is obvious. If $u \notin W$, write

$$
u=u^{\prime} a b^{2} u^{\prime \prime}
$$

with $u^{\prime \prime}$ as short as possible, so that

Choose $k$ so that

$$
u \psi=u^{\prime} b a b a u^{\prime \prime} .
$$

$$
\left(u^{\prime \prime} v\right) \psi^{k+1}=\left(u^{\prime \prime} v\right) \psi^{k}=w,
$$

say, so that $w \in W$; and choose $k$, moreover, as small as consistent with this. Thus $k=0$ if $u^{\prime \prime} v \in W$, and otherwise $k$ is such that $\left(u^{\prime \prime} v\right) \psi^{k-1} \notin W$. Now

$$
\begin{aligned}
((u \psi) v) \psi^{k} & =\left(u^{\prime} b a b a u^{\prime \prime} v\right) \psi^{k} \\
& =u^{\prime} b a b a\left(u^{\prime \prime} v\right) \psi^{k}=u^{\prime} \text { babaw }
\end{aligned}
$$

where we have used the minimality of $k$. Also

$$
\begin{aligned}
(u v) \psi^{k+1} & =\left(u^{\prime} a b^{2} u^{\prime \prime} v\right) \psi^{k+1} \\
& =\left(u^{\prime} a b^{2}\left(u^{\prime \prime} v\right) \psi^{k}\right) \psi=\left(u^{\prime} a b^{2} w\right) \psi \\
& =u^{\prime} b a b a w
\end{aligned}
$$

and (4.51) follows. This completes the proof of Lemma 4.2.

From now on we refer to the normal word that represents an element as its normal form.

\section{Proof of the rigidity}

In order to complete the proof of Theorem 3.1, we consider an endomorphism $\eta: A \rightarrow A$ given by

$$
\begin{aligned}
& a \eta=u, \\
& b \eta=v,
\end{aligned}
$$

where we may assume that $u$ and $v$ are given in normal form. We must then have

$$
u v^{2}=v u v u,
$$

and this must be a consequence of the defining relation

$$
a b^{2}=b a b a
$$

of $A$. We first remark that $u$ is a power of $a$, say

$$
u=a^{p}, \quad p \geqq 1 ;
$$

for application of the defining relation leaves the total exponent of $b$ un- 
changed, while $u$ appears with different exponents on the two sides of (5.1).

The argument now consists in considering the various forms that $v$ can have, reducing both $u v^{2}$ and vuvu to normal form, and showing that (5.1) is not satisfied except trivially. We assume that $v$ is given in the form

$$
v=b^{t} a^{s_{1}} b a^{s_{2}} \cdots b a^{s_{n}}
$$

subject to $(4.12-4.14)$.

Now if $n \geqq 2$, then the reduction to normal form of $u v^{2}$ will not affect the part $b a^{s_{2}} \cdots b a^{s_{n}}$ with which the second $v$ ends; thus the normal form of $u v^{2}$ ends in $a^{s_{n}}$. Similarly the end of the second $v$ will not be affected by the reduction of vuvu to normal form; thus this normal form will end in $a^{\boldsymbol{s}_{n}+\boldsymbol{p}}$. These normal forms then are manifestly different.

Consider then $n=1$ and $s_{1}>0$. Now the normal form of

$$
u v^{2}=a^{p} b^{t} a^{s_{1}} b^{t} a^{s_{1}}
$$

will end in $a^{s_{1}}$ if $t$ is odd, in $a^{s_{1}+1}$ if $t$ is positive and even, and in $a^{p+2 s_{1}}$ if $t=0$; while the normal form of

$$
v u v u=b^{t} a^{s_{1}+p} b^{t} a^{s_{1}+p}
$$

will end in $a^{s_{1}+p}$ if $t$ is odd, in $a^{s_{1}+p+1}$ if $t$ is positive and even, and in $a^{2 s_{1}+2 p}$ if $t=0$ : and again these normal forms are different.

Thus we may now assume that $n=1$ and $s_{1}=0$, that is to say $v=b^{t}$; and as $v$ cannot be the empty word, we must now have $t>0$. Now the normal form of

$$
u v^{2}=a^{p} b^{2 t}
$$

will end in $a$, while the normal form of

$$
v u v u=b^{t} a^{p} b^{t} a^{p}
$$

will end in $a^{p}$ or $a^{p+1}$ according as $t$ is odd or even. These normal forms then cannot be equal unless $p=1$, that is $u=a$. In this case, if $t>1$, then the normal form of

$$
u v^{2}=a b^{2 t}
$$

is

while the normal form of

$$
a b^{2 t}=b^{2}(a b)^{2 t-3} a^{2} b a,
$$

$$
v u v u=b^{t} a b^{t} a
$$

begins with $b^{t+1}$ or even, if $t>3$, with $b^{t+2}$. Thus these normal forms are again different, and we are left only with the case $t=1$, that is to say $v=b$. Hence $\eta$ is the identity on $A$, and the proof of the theorem is complete. 
[Added 25 March 1968.] The existence of rigid semigroups also follows from Proposition 4 in a paper by Zdeněk Hedrlín and Joachim Lambek, 'How comprehensive is the category of semigroups?', submitted to the Journal of Algebra. We are grateful to Dr Hedrlín and Professor Lambek for allowing us to see a preprint of their paper. They are concerned with rather more general problems than we, and their methods are quite different from ours.

\section{References}

[1] Reinhold Baer, 'Finite extensions of abelian groups with minimum condition', Trans. Amer. Math. Soc. 79, (1955) 521-540.

[2] Reinhold Baer, 'Kriterien für die Endlichkeit von Gruppen', Jber. Deutsch. Math.-Verein. 63, (1960) 53-77.

[3] A. H. Clifford and G. B. Preston, The algebraic theory of semigroups, volume II, (Amer. Math. Soc., Providence, R.I. 1967).

[4] W. Ledermann and B. H. Neumann, 'On the order of the automorphism group of a finite group. I', Proc. Roy. Soc. London, A, 233, (1956) 494-506.

[5] B. H. Neumann, 'Some remarks on semigroup presentations', Canad. J. Math. 19, (1967) 1018-1026.

The Australian National University

Canberra 